\title{
Automated Rapid Preparation of Tissue Specimens for TEM Pathology
}

\author{
Thomas E Strader ${ }^{1}$, Nicholas R Stewart ${ }^{1,2}$, Benjamin K August ${ }^{1}$, Steven L Goodman ${ }^{1}$ \\ ${ }^{1 .}$ Microscopy Innovations, LLC, Marshfield, USA. \\ 2. University of Wisconsin-Madison School of Medicine and Public Health, Madison, USA.
}

The typical time to prepare clinical tissue specimens for TEM varies from 1-2 days to several hours, with microwave processing heretofore providing the most rapid preparation [1,2]. Microwave processing is accomplished by the manual exchange of each processing reagent alternated by placing the reagent-immersed specimens into a microwave oven. Following the final resin infiltration step, each tissue samples is then manually transferred to an embedding mold for polymerization. This manual process requires the full attention of the microscopist for the entire protocol followed by an hour to transfer specimens to embedding molds [2]. In the present study, mammalian tissues were automatically prepared for TEM in 2 hours or less (prior to resin curing) with an ASP-1000 Automatic Specimen Processor. Rather than microwaves, the ASP used rapid mixing to accelerate processing by enhancing reagent diffusion. Specimens remained in the same labeled mPrep capsule from initial fixation through sectioning, thus eliminating tedious manual transfers to embedding molds and ensuring continuous specimen labeling.

Mouse tissues were excised, cut into 1-2 mm pieces, inserted into $\mathrm{mPrep} / \mathrm{s}$ capsules and fixed overnight at $5 \mathrm{C}$ in phosphate buffered $4 \%$ paraformaldehyde and $1 \%$ glutaraldehyde. Rats were perfusion-fixed, tissues were excised and then subsequently handled as per mouse tissues. Specimens in $\mathrm{mPrep} / \mathrm{s}$ capsules were attached to the ASP and processed with a conventional chemical protocol of $1 \%$ phosphate buffered $\mathrm{OsO}_{4}$, water washes, graded ethanols, acetone, and Embed 812. Rapid processing was achieved with reagent exchanges as fast as to 2 per second, and 600 exchanges for $\mathrm{OsO}_{4}$ and 180 exchanges for each resin infiltration step. $\mathrm{mPrep} / \mathrm{s}$ capsules with $100 \%$ resin infiltrated specimens were then removed from the ASP for overnight polymerization at $60^{\circ} \mathrm{C}$. Following polymerization, capsules were directly mounted in the microtome, thus reducing effort, preserving orientation and maintaining specimen labeling. $70 \mathrm{~nm}$ sections were UA and lead stained for imaging at $80 \mathrm{KeV}$ with a Philips CM120.

Kidney was processed in 45 minutes prior to resin curing (Figure 1), while skeletal muscle, liver, brain and heart (Figures 2-5) were processed in 133 minutes. Tissues were well-preserved with no evidence of incomplete processing as seen with TEM or light microscopy (not shown). Consistency was demonstrated with 24 well-prepared liver specimens prepared in 3 ASP processing batches (Figure 3b).

ASP sample preparation speed was comparable to manual microwave preparation but was much easier since processing was entirely automatic. Further, since specimens were never removed from $\mathrm{mPrep} / \mathrm{s}$ capsules, this eliminated messy, time-consuming handling in resin and the potential for mix-up errors. Although overnight resin polymerization was used in this study, high temperature thermal or microwave curing could further reduce total preparation time. Rapid aldehyde initial fixation can also be done with the ASP when specimens are not provided to the lab already immersed in a primary fixative.

References

[1] Giberson RT et al, Ultrastructural Pathology 27 (2003) p. 187. 
[2] Schroeder JA et al, Micron 37 (2006) p. 577.
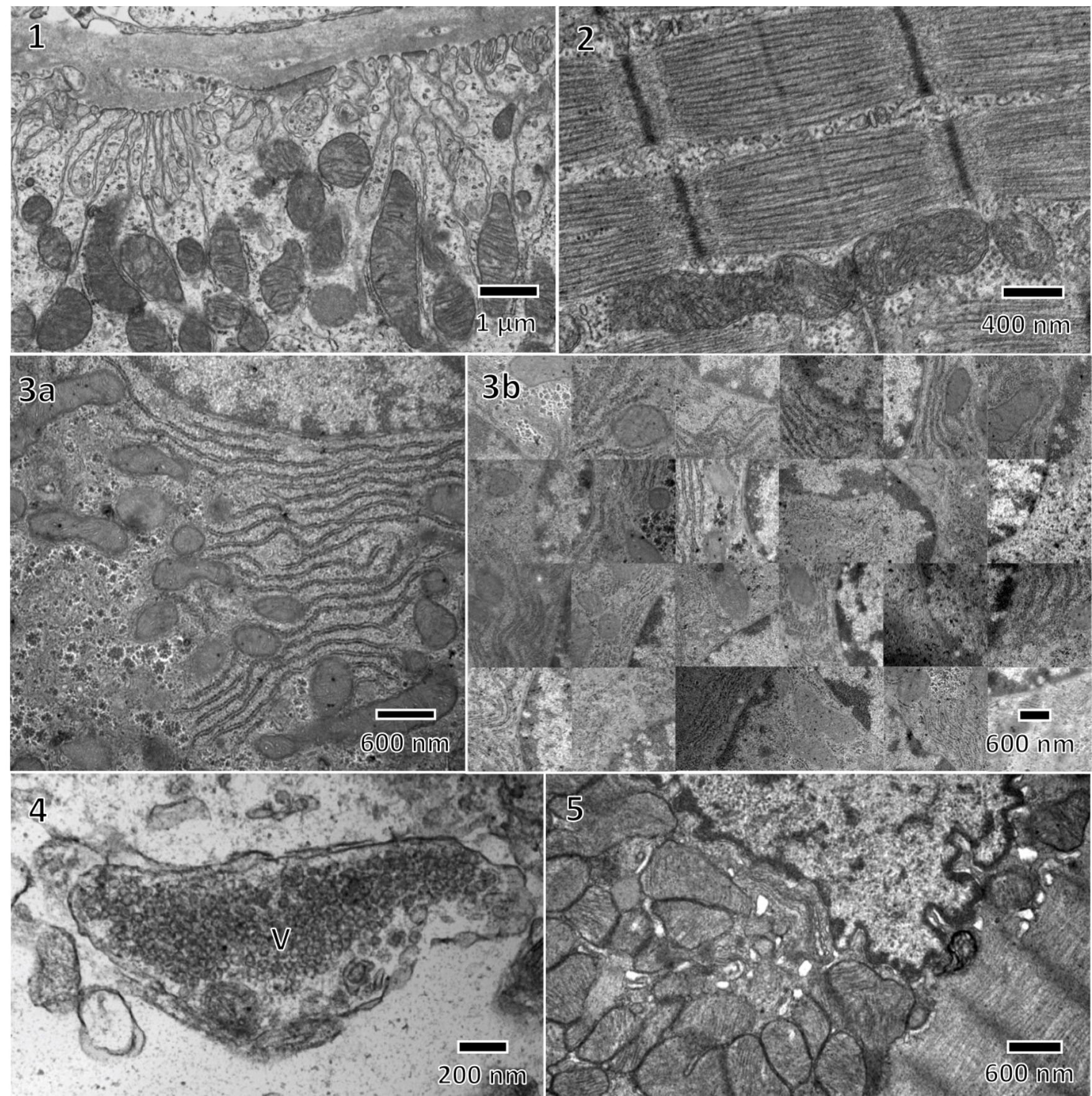

Figure 1: Kidney (rat) proximal convoluted tubule with invaginating membrane folds on basolateral side. Figure 2: Gastrocnemius skeletal muscle (rat) myofibril with sarcomeres, mitochondria sarcoplasmic reticulum, Z-lines, and M-lines. Figure 3: Liver hepatocyte (rat) with clear rough endoplasmic reticulum, glycogen particles, mitochondria and a nucleus (a). Reproducibility shown with 24 liver samples (b). Figure 4: Brain synapse (mouse) with synaptic vesicles (V). Figure 5: Cardiac muscle (mouse). 